\title{
Imunodeficiência comum variável: diagnóstico tardio em adulto com manifestação sinopulmonar
}

\author{
Variable common immunodeficiency: late diagnosis in adults with synopulmonary \\ manifestation \\ Inmunodeficiencia comun variable: diagnóstico tardío en adulto con manifestación \\ sinopulmonar
}

\begin{abstract}
Paula Scatolino Silva ${ }^{1 *}$, Paloma Duarte Motta $^{1}$, Raphaela Fabri Viana do Nascimento ${ }^{1}$, Sarah Teixeira Dantas ${ }^{1}$, Larissa Veiga Raña ${ }^{1}$, Jorge Henrique Fares Depieri ${ }^{1}$, Thereza Drummond Campos $^{1}$, Fernanda Ferreira Bicalho Moreira ${ }^{1}$, Leonardo Meira de Faria ${ }^{2}$, Lourenço Gonçalves Ferreira ${ }^{1}$.
\end{abstract}

\section{RESUMO}

Objetivo: Relatar um caso de diagnóstico tardio de imunodeficiência comum variável, em paciente da terceira idade, com histórico de infecções sinopulmonares recorrentes. Detalhamento do caso: Paciente feminino, 63 anos, engenheira civil, portadora de bronquiectasia. Admitida com quadro de tosse produtiva e piora da dispneia basal iniciados há dois dias. À admissão, exames revelando anemia, leucocitose importante, plaquetose e proteína $C$ reativa elevada. Histórico de infecções de vias aéreas de repetição de difícil tratamento. Iniciou-se investigação de possíveis causas da doença estrutural pulmonar (bronquiectasia): exames de eletroforese de proteínas com presença de hipogamaglobulinemia, além de baixa dosagem de IgA e IgM, confirmando a hipótese de imunodeficiência comum variável. Iniciado tratamento com imunoglobulina endovenosa. Recebeu alta hospitalar assintomática, com programação de seguimento ambulatorial e tratamento com imunoglobulinas a cada 28 dias. Não recorreu com infecção sinopulmonar no período de um ano após alta hospitalar. Considerações finais: A imunodeficiência deve ser considerada como diagnóstico diferencial em pacientes com infecções sinopulmonares recorrentes. O diagnóstico precoce permite o tratamento imediato, diminuindo a morbidade e proporcionando melhor qualidade de vida.

Palavras-chave: Imunodeficiência comum variável, Adulto, Sinopulmonar.

\begin{abstract}
Objective: Report a case of late diagnosis of variable common immunodeficiency, in an elderly patient, with a history of recurrent sinopulmonary infections. Case description: Female patient, 63 years old, civil engineer, with bronchiectasis. Admitted with a productive cough and worsening of baseline dyspnea started two days before medical care. Upon admission, tests showing anemia, significant leukocytosis, platelets and elevated C-reative protein. History of recurrent refractory airway infections, difficult to treat. The investigation of possible causes of structural lung disease (bronchiectasis) began: exams of protein electrophoresis with the presence of hypogammaglobulinemia, in addition to low dosage of $\lg \mathrm{A}$ and $\operatorname{lgM}$, confirming the hypothesis of common variable immunodeficiency, therefore starting treatment with intravenous immunoglobulin. She was discharged asymptomatic, with an outpatient follow-up schedule and treatment with immunoglobulins every 28 days. She did not recur with sinopulmonary infection within one year after hospital discharge. Final considerations: Immunodeficiency should be considered as a differential diagnosis in patients with recurrent sinopulmonary infections. Early diagnosis allows for immediate treatment, decreasing assimilation to morbidity and providing better quality of life.
\end{abstract}

Key words: Common variable immunodeficiency, Adult, Sinopulmonary.

\footnotetext{
${ }^{1}$ Hospital Felício Rocho (HFR) em parceria com a Pós-Graduação Ciências Médicas - Minas Gerais (PGCMMG), Belo Horizonte - MG. *E-mail: paulascatolinos@yahoo.com.br

2 Universidade Federal de Minas Gerais, Belo Horizonte - MG.
}

SUBMETIDO EM: 2/2021

ACEITO EM: 2/2021

PUBLICADO EM: 3/2021 


\section{RESUMEN}

Objetivo: Relatar un caso de diagnóstico tardío de inmunodeficiencia común variable en paciente de la tercera edad, con histórico de infecciones sinopulmonares recurrentes. Detalles del caso: Paciente femenino, 63 años, ingeniera civil, portadora de bronquiectasia. Hospitalizada con cuadro de tos productiva y empeoramiento de la disnea basal iniciado hace dos días. Al ingreso, exámenes mostrando anemia, leucocitosis importante, trombocitosis y proteína $C$ reactiva elevada. Antecedente de infecciones de vías aéreas a repetición, de difícil manejo. Se comenzó investigando posibles causas de la enfermedad estructural pulmonar (bronquiectasia): electroforesis de proteínas con presencia de hipogammaglobulinemia, además de bajos niveles de IgA e IgM; confirmando la hipótesis de Inmunodeficiencia común variable. Fue iniciado tratamiento con inmunoglobulina intravenosa. Recibió alta hospitalar, asintomática; con seguimiento por consulta externa y tratamiento con inmunoglobulinas cada 28 días. No presentó recurrencia de infecciones sinopulmonares en el período de un año después de recibir alta. Consideraciones finales: La inmunodeficiencia común variable debe ser considerada como diagnóstico diferencial em pacientes con infecciones sinopulmonares recurrentes. El diagnóstico precoz permite un tratamiento inmediato, disminuyendo la mortalidad y mejorando la calidad de vida.

Palabras clave: Inmunodeficiencia común variable, Adulto, Sinopulmonar.

\section{INTRODUÇÃO}

A imunodeficiência comum variável (CVID) é compreendida como um grupo de distúrbios hipogamaglobulinêmicos com apresentações fenotípicas heterogêneas. É caracterizada por hipogamaglobulinemia, produção diminuída de anticorpos específicos e células $B$ defeituosas, sem causa estabelecida (GEREIGE JD e MAGLIONE PJ, 2019; YAZDANI R, et al., 2020).

É considerada a imunodeficiência humoral mais comumente diagnosticada em adultos, com incidência variando de 1:25.000-50.000 casos na população geral. A ocorrência da doença é semelhante entre o sexo feminino e masculino, e a média do diagnóstico ocorre na faixa etária entre 20 a 40 anos (BERRON-RUIZ L, et al., 2018; CUNNINGHAM-RUNDLES C, 2019).

A deficiência na imunidade humoral leva a uma maior suscetibilidade a infecções sinopulmonares e à formação de bronquiectasias, considerada a complicação mais comum - uma média de $27-34 \%$ dos pacientes são acometidos. Cerca de $80 \%$ dessas infecções pulmonares são causadas por microrganismos encapsulados, como Streptococcus pneumoniae, Haemophilus influenza, Staphylococcus aureus e Pseudomonas aeruginosa (associada a um pior prognóstico). A desregulação imune aumenta a predisposição a malignidade, alergias, doenças autoimunes, doenças granulomatosas e enteropatias, além de acarretar uma baixa resposta aos protocolos de imunização (DIVINO PHA, et al., 2015; AZIZI G, et al., 2016).

Os critérios diagnósticos evoluíram desde que foi feito o primeiro diagnóstico de exclusão em 1971 pela Organização Mundial da Saúde. Um consenso internacional de 2016 sobre CVID definiu: níveis séricos reduzidos de imunoglobulinas $\lg \mathrm{G}$, IgA ou $\lg \mathrm{M}$, e baixa resposta de anticorpos à vacinação em um indivíduo com pelo menos 4 anos de idade, sem causa secundária conhecida de hipogamaglobulinemia. Os critérios da Sociedade Europeia de Imunodeficiências diferem desses por exigirem a presença de sintomas como aumento de infecções, manifestações autoimunes, doenças granulomatosas e linfoproliferativas policlonais inexplicadas, além das anormalidades laboratoriais citadas acima, para fechar o diagnóstico de CVID (GEREIGE JD e MAGLIONE PJ, 2019; YAZDANI R, et al., 2020).

$O$ atraso no diagnóstico é comum, podendo ocorrer até 15 anos após o início dos sintomas. Acredita-se que devido a este atraso, a medicina interna raramente as aborda, o que contribui para um pior prognóstico, aumento de comorbidades e aumento do custo para o sistema de saúde (CUNNINGHAM-RUNDLES C, 2019; MODELL V, et al., 2016).

O tratamento para pacientes com CVID inclui a administração de terapia de reposição de imunoglobulina humana e o combate a infecções, normalmente com uso de antibioticoterapia contra germes encapsulados. As imunoglobulinas contêm anticorpos neutralizantes contra uma ampla variedade de bactérias e vírus, 
estimulando a memória imunológica dos doadores. Devem ser administradas a cada três ou quatro semanas. Dados de uma metanálise mostraram redução de $27 \%$ na incidência de pneumonia para cada aumento de $100 \mathrm{mg} / \mathrm{dL}$ no nível de IgG sérica nos pacientes com deficiência de anticorpos (DIVINO PHA, et al., 2015; NOBRE FA, et al., 2016).

A antibioticoterapia profilática para redução de infecções respiratórias não está claramente estabelecida; no entanto, recomenda-se o uso de macrolídeos e quinolonas para este fim. Embora as infecções oportunistas por Pneumocystis jiroveci e Cytomegalovirus não sejam características, elas podem ser encontradas em um subgrupo de pacientes com baixa contagem de células T CD4. Para esses casos, recomenda-se a profilaxia com trimetoprim / sulfametoxazol (YAZDANI R, et al., 2020; GATHMANN B, et al., 2014).

Esse artigo tem como objetivo descrever um caso clínico de diagnóstico tardio de imunodeficiência comum variável, em paciente do sexo feminino, 63 anos, com histórico de infecções sinopulmonares recorrentes, associada a bronquiectasia, na qual foi detectada hipogamaglobulinemia grave. Ainda, trazer para a ciência mais dados sobre a doença, as alterações encontradas nos exames laboratoriais que levaram ao diagnóstico e a experiência com o início do tratamento.

\section{DETALHAMENTO DO CASO}

O presente artigo foi conduzido como estudo observacional do tipo relato de caso, submetido à Plataforma Brasil e aprovado pelo Comitê de Ética em Pesquisa com Seres Humanos (CAAE 17009519.2.0000.5125).

Paciente de 63 anos, sexo feminino, engenheira civil, solteira, branca, natural e residente em Belo Horizonte-MG. Admitida em unidade hospitalar em maio de 2019, apresentando há dois dias da admissão quadro de tosse produtiva, piora da dispneia basal, palpitações e mal-estar. Paciente sabidamente portadora de bronquiectasia, diagnosticada em 2014.

Ao exame físico, encontrava-se em regular estado geral, subfebril, hipertensa (PA 200x100 mmHg), taquicárdica (FC $130 \mathrm{bpm}$ ), taquipneica (FR 24irpm), com queda importante da saturação de oxigênio (SpO2 $79 \%$ em ar ambiente). À ausculta respiratória, crepitações difusas bilateralmente. Os exames laboratoriais permitiram identificar a presença de anemia ( $\mathrm{Hb} 10,1)$, leucocitose importante (global de leucócitos de 24800), plaquetose (plaquetas 861.000 ) e aumento de proteína C reativa (99).

Quanto a história patológica pregressa, foi submetida a tireoidectomia em 2002 e nefrectomia a esquerda em 2012, por doença oncológica nestes sítios. Além disso, a partir de 2014, refere início de quadro de infecções de vias aéreas superiores (rinossinusite) e inferiores (pneumonia) de repetição e de difícil tratamento, com uso de inúmeros esquemas de antibióticos, por vezes demandando internações em unidade hospitalar.

Recebeu o diagnóstico de bronquiectasia ao final de 2014, em uma de suas internações, porém sem investigação da causa. Refere ainda exame de colonoscopia realizada em $2018 \mathrm{com}$ achado de pólipo séssil, enviado material para análise histopatológica. Afirmou que durante a infância e grande parte da vida adulta não havia relato de recorrência de infecções nesta época. Fazia uso regular de levotiroxina 150mcg/dia. Paciente nega tabagismo, relato de etilismo social.

Foi realizado exame tomográfico do tórax que evidenciou aumento do calibre dos brônquios, áreas de bronquiectasia de morfologia cilíndrica, em que ocorre dilatação uniforme dos brônquios segmentares com predomínio nos lobos inferiores e médio, associado a um importante espessamento das paredes das vias respiratórias. Visualizado também acúmulo de secreção mucoide dentro das áreas de bronquiectasia, formando áreas de opacidade centrolobulares ramificadas ou aspecto de "árvore em brotamento". Na imagem é possível identificar essas alterações através das setas pretas (Figura 1). 
Figura 1 - Tomografia de tórax com áreas de bronquiectasia cilíndricas. Imagem com aumento do calibre dos brônquios e espessamento significativo de suas paredes em lobos inferiores e médio (setas pretas); presença de atelectasia (seta vermelha) e consolidação do parênquima pulmonar sugestivo de infecção (seta verde).

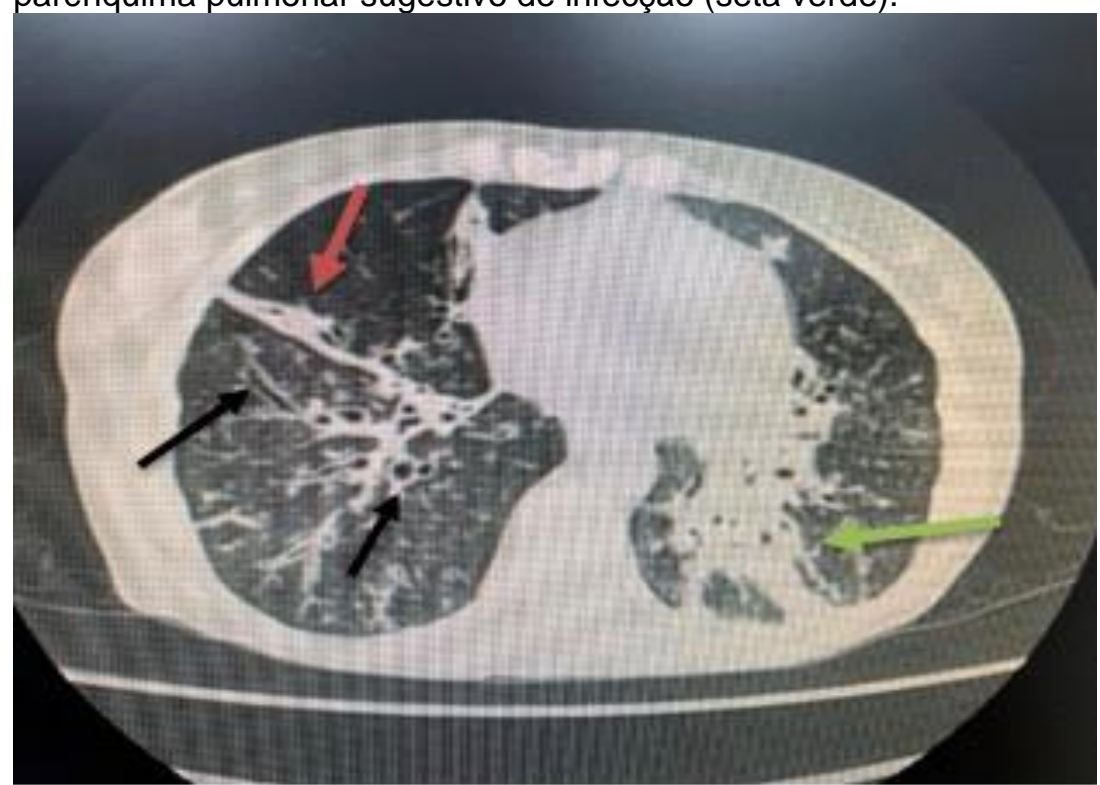

Fonte: Silva PS, et al., 2021.

$\mathrm{Na}$ tomografia de seios da face é possível identificar através das setas vermelhas espessamento do revestimento mucoso dos seios da face evidenciando pansinusopatia (Figura 2). Há indícios de cirurgia prévia de abertura do seio maxilar para tratamento de sinusite crônica-antrostomia.

Figura 2 - Tomografia de seios da face com pansinusopatia e antrostomia bilateral. Imagem com espessamento do revestimento mucoso dos seios frontal, etmoidal e antros maxilares (setas vermelhas), com preenchimento de material de densidade em partes moles em seio esfenoidal e em células etmoidais.

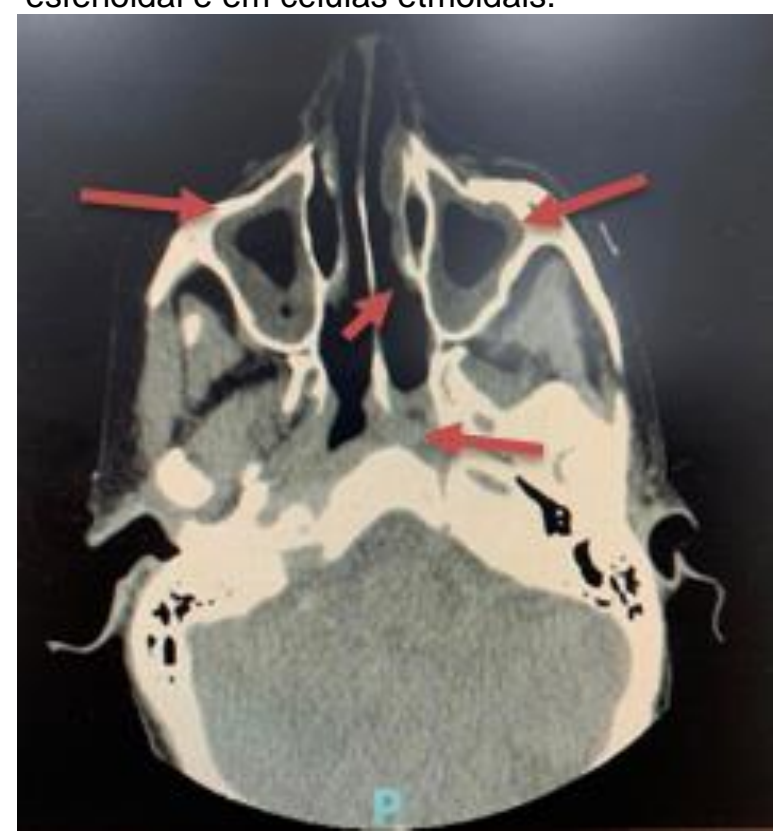

Fonte: Silva PS, et al., 2021. 
Iniciado tratamento para bronquiectasia de forma difusa infectada com esquema de antibiótico intravenoso de largo espectro com cobertura para pseudomonas por 14 dias, associado a medidas para higienização broncoalveolar com micronebulização com brometo de ipratrópio, em seguida gentamicina inalatória e, por último, salina hipertônica. Além disso, foi instituído tratamento para pansinusopatia com corticoide tópico e lavagem nasal. Paciente apresentou melhora significativa do quadro inicial.

Durante internação, iniciou-se investigação de possíveis causas e fatores predisponentes para o desenvolvimento de doença estrutural pulmonar. Paciente apresentava exames externos, que já sinalizavam para comprometimento de resposta imunológica humoral, dentre eles: eletroforese de proteínas com presença de hipogamaglobulinemia gama 0,07 (Valor de referência $0,7-1,7$ ), dosagem de $\lg A<33$ (Valor de referência $40-350$ ), e $\lg E<1,0$ (Valor de referência até 87 ).

Diante da história clínica e exames prévios, foram realizados novos exames para extensão propedêutica e, após descartadas outras causas de imunodeficiência, foi confirmada a hipótese de CVID. Entre os principais exames laboratoriais destacam-se: sorologia para o vírus da imunodeficiência humana (HIV) I e II e provas reumatológicas negativas; imunoglobulinas $\lg \mathrm{A}$, $\lg \mathrm{M}$ e todas as subclasses de $\lg \mathrm{G}$ reduzidas, e linfócitos $\mathrm{B}$ diminuídos, como ilustrados na tabela abaixo (Tabela 1).

Tabela 1 - Exames laboratoriais com redução significativa de todas as imunoglobulinas.

\begin{tabular}{|c|c|c|c|}
\hline \multicolumn{2}{|c|}{ Exame laboratorial } & Resultado & Valor de referência \\
\hline \multicolumn{2}{|c|}{ Imunoglobulina IgA } & $23 \mathrm{mg} / \mathrm{dL}$ & 40 a $350 \mathrm{mg} / \mathrm{dL}$ \\
\hline \multicolumn{2}{|c|}{ Imunoglobulina IgM } & $8 \mathrm{mg} / \mathrm{dL}$ & 50 a $300 \mathrm{mg} / \mathrm{dL}$ \\
\hline \multicolumn{2}{|c|}{ Imunoglobulina IgG } & $70 \mathrm{mg} / \mathrm{dL}$ & 650 a $1600 \mathrm{mg} / \mathrm{dL}$ \\
\hline \multirow{4}{*}{$\begin{array}{c}\text { Subclasses de } \\
\lg G\end{array}$} & $\lg G 1$ & $110 \mathrm{mg} / \mathrm{L}$ & 3422 a $11178 \mathrm{mg} / \mathrm{L}$ \\
\hline & $\lg 2$ & $93 \mathrm{mg} / \mathrm{L}$ & 1476 a 5246 mg/L \\
\hline & $\lg G 3$ & $14 \mathrm{mg} / \mathrm{L}$ & 211 a $1142 \mathrm{mg} / \mathrm{L}$ \\
\hline & $\operatorname{lgG} 4$ & $3 \mathrm{mg} / \mathrm{L}$ & 69 a $888 \mathrm{mg} / \mathrm{L}$ \\
\hline
\end{tabular}

Fonte: Silva PS, et al., 2021.

Diante do diagnóstico de imunodeficiência comum variável, foi iniciado tratamento com imunoglobulina endovenosa. Paciente recebeu alta hospitalar assintomática, estável do ponto de vista clínico e hemodinâmico, com programação de seguimento em uso de imunoglobulinas a cada 28 dias. Em acompanhamento ambulatorial, paciente não recorreu com infecção sinopulmonar no período de um ano de observação após alta hospitalar.

\section{DISCUSSÃO}

Pacientes com CVID apresentam uma ampla gama de manifestações clínicas, incluindo infecções bacterianas recorrentes, doenças autoimunes, doenças pulmonares e do trato gastrointestinal, doenças linfoproliferativas, malignidade e doenças alérgicas. A heterogeneidade clínica, leva a desafios de diagnóstico, sendo necessário afastar outras causas de hipogamaglobulinemia secundária, como perdas gastrointestinais (enteropatia perdedora de proteínas), síndrome nefrótica, neoplasias hematológicas, infecções virais e uso de medicação (imunossupressores e quimioterapia). Com isso torna-se importante lembrar que o diagnóstico da doença é de exclusão (YAZDANI R, et al., 2020; CUNNINGHAM-RUNDLES C, 2019).

A paciente do caso exposto se enquadrava nesse amplo espectro clinico pela história de tumor renal e na tireoide, doença poliposa intestinal e pneumonia de repetição por pseudomonas, atendendo ao critério clínico de aumento de suscetibilidade a infecções pulmonares. Quanto ao critério laboratorial, a eletroforese de proteínas com presença de hipogamaglobulinemia, além de baixa dosagem de $\operatorname{lgA}$ e $\operatorname{lgM}$, foram dados relevantes no diagnóstico. Ao exame de imagem, a TC de tórax foi essencial ao evidenciar bronquiectasia cilíndricas que foram correlacionadas com sequelas pulmonares devido às infecções de repetição.

Existem fortes evidências de fatores genéticos relacionados a apresentação e progressão da doença, através de mutações deletérias em diferentes genes. Um esquema de classificação com base nos fenótipos 
de células $B$ de memória, em que pacientes foram randomizados em três grupos com as seguintes características: ausência de produção de células $B$, apenas produção de $\lg M$ ou produção normal de $\lg M$ e IgG, não se mostrou eficaz. Ainda, a avaliação de subconjuntos de células $\mathrm{B}$, como análise de contagem total e de células $B$ de transição, também não trouxe benefício. Observou-se que os pacientes podem migrar entre as classificações ao longo do tempo, o que não permite intervenção especifica (ABBOTT JK e GELFAND EW, 2015).

Há ainda uma possível divisão dos pacientes em subgrupos de complicações da doença, que se mostrou favorável em relação à desfechos de melhor prognóstico, uma vez que percebeu-se ser uma classificação estável e passível de intervenção. As quatro categorias definidas foram: nenhuma complicação relacionada a doença, presença de citopenias, presença de linfoproliferação policlonal e enteropatia persistente. As três últimas foram relacionadas a piora de sobrevida (ABBOTT JK e GELFAND EW, 2015).

Em 2019, a Sociedade de Imunologia do Reino Unido elaborou um consenso de recomendações para tratamento e monitoramento de complicações não infecciosas da CVID, devido ao baixo número de evidencias na área. O conhecido aumento do risco de malignidade nesses pacientes, levaram especialistas a uma definição sobre avaliação clínica regular e exames de sangue e de imagem para monitoramento.

Dentre as recomendações citadas no consenso, chama atenção a importância da pesagem regular a cada seis meses e palpação de linfonodos e abdome a cada um ano para monitoramento de linfadenopatias e presença de massas palpáveis. Nos exames de sangue, há indicação de hemograma completo semestral para monitorar desenvolvimento de citopenais autoimunes e rastreio de anemias com dosagem do nível sérico de vitamina B12, folato e ferritina. Entre os exames de imagem, a tomografia de tórax de alta resolução deve ser realizada a cada cinco anos para avaliar o desenvolvimento ou progressão de bronquiectasia naqueles pacientes com infecções pulmonares de repetição. Ressalta-se também a importância de uma abordagem multidisciplinar (BETHUNE C, et al., 2019).

A terapia de reposição de imunoglobulina humana endovenosa é atualmente a base do tratamento para pacientes com CVID. As reações adversas à sua administração, locais ou sistémicas, podem ocorrer em diferentes graus de gravidade, mas na sua maioria não implicam na suspensão do tratamento, sendo necessária substituição para administração subcutânea. $O$ transplante de células-tronco hematopoiéticas pode ser uma opção viável para pacientes que tenham defeitos imunológicos celulares e autoimunidade resistente à terapia. Poucos dados estão disponíveis sobre as vantagens de novas técnicas de imunomodulação (STIEHM ER, 2013; YAZDANI R, et al., 2020).

Os pacientes com CVID apresentam um risco maior de mortalidade. E ocorrem principalmente por causas malignas, doença pulmonar crônica, infecção e hepatopatias. Estudos retrospectivos conseguiram atribuir esse aumento principalmente às complicações não infecciosas da doença, em comparação com às infecciosas. Apesar da sua redução com o avanço do tratamento, o risco de mortalidade por complicações não infecciosas ainda é significativa e pode ocorrer de forma precoce (ABBOTT JK e GELFAND EW, 2015).

Desde a adoção do tratamento, a mortalidade desses pacientes diminuiu de 30\% no início de 1990 para $15 \%$ no início de 2000 , segundo um coorte de 240 pacientes no Reino Unido, que foram seguidos por cerca de duas décadas. A melhora na sobrevida foi atribuída à redução de complicações infecciosas após reposição de imunoglobulina e melhores drogas antimicrobianas. Embora a sobrevida geral tenha melhorado, os pacientes continuam a ter sobrevida reduzida em comparação com outros de mesma idade. As manifestações não infecciosas como as doenças autoimunes, linfoproliferativas e doenças pulmonares crônicas, não são melhoradas apenas com o uso de imunoglobulina (GEREIGE JD e MAGLIONE PJ, 2019).

Apesar da resposta satisfatória inicial da paciente à terapia com imunoglobulinas, a mesma foi referenciada a um centro especializado em imunologia para acompanhamento e reavaliação frequente de indicação de novas alternativas de tratamento. Ao referenciar a paciente dos cuidados hospitalares para unidades especializadas de forma precoce, foi possível maior auxílio na condução da doença, com provável melhor desfecho. A melhora clinica obtida se alinha aos estudos que mostram que alvos mais altos nas concentrações de IgG após reposição de imunoglobulinas são especialmente importantes em pacientes com doença pulmonar crônica e bronquiectasia (TRINCÃO DP, et al., 2020; GOUDOURIS ES, et al., 2017). 
Diante do exposto, a imunodeficiência deve ser sempre considerada como diagnóstico diferencial por profissionais de saúde que tratam pacientes com infecções sinopulmonares recorrentes. O rastreio básico com dosagem de imunoglobulinas deve ser realizado nesses pacientes. O diagnóstico precoce permite 0 tratamento imediato, diminuindo assim a morbidade e proporcionando melhor qualidade de vida. Dessa forma, o caso apresentado serve como alerta para médicos não especialistas, possibilitando intervenção terapêutica e manejo adequado precocemente, além de possível referenciamento de pacientes para centros especializados.

\section{REFERÊNCIAS}

1. ABBOTT JK, GELFAND EW. Common Variable Immunodeficiency: Diagnosis, Management, and Treatment. Immunol Allergy Clin North Am. 2015; 35(4): 637-58.

2. AMERATUNGA R, WOON ST. Perspective: Evolving Concepts in the Diagnosis and Understanding of Common Variable Immunodeficiency Disorders (CVID). Clin Rev Allergy Immunol. 2020; 59(1): 109-121.

3. AZIZI G, et al. Autoimmunity in common variable immunodeficiency: epidemiology, pathophysiology and management. Expert Review of Clinical Immunology, 2017; 13(2): 101-115.

4. BERRON-RUIZ L, et al. Inmunodeficiencia común variable y su asociación con defectos en células B de memória. Rev. alerg. Méx, 2018, 65(2): 171-177.

5. BETHUNE C, et al. British Society for Immunology/United Kingdom Primary Immunodeficiency Network consensus statement on managing non-infectious complications of common variable immunodeficiency disorders. Clin Exp Immunol. 2019; 196(3): 328-335.

6. BUENO J, FLORS L. Papel de los estudios de imagen en el diagnóstico etiológico de las bronquiectasias: la distribución es la clave. Radiología. 2018; 60(1): 39-48.

7. CUNNINGHAM-RUNDLES C. Common variable immune deficiency: Dissection of the variable. Immunol Rev. 2019; 287(1): 145-161.

8. CUNNINGHAM-RUNDLES C. Common variable immune deficiency: case studies. Blood. 2019; 134(21): 1787-1795.

9. DIVINO PHA, et al. Bronchiectasis caused by common variable immunodeficiency. J. bras. Pneumol., 2015; 41(5): 482-483.

10. GATHMANN B, et al. Clinical picture and treatment of 2212 patients with common variable immunodeficiency. J Allergy Clin Immunol. 2014; 134(1): 116-126.

11. GEREIGE, JD, MAGLIONE PJ. Current Understanding and Recent Developments in Common Variable Immunodeficiency Associated Autoimmunity. Front Immunol. 2019; 10: 2753.

12. GOUDOURIS ES, et al. II Brazilian Consensus on the use of human immunoglobulin in patients with primary immunodeficiencies. 2017; 15(55 21): 1-16.

13. MODELL V, et al. Primary immunodeficiencies worldwide: an updated overview from the Jeffrey Modell Centers Global Network. Immunol Res. 2016; 64(3): 736-753.

14. MUŞABAK UH, et al. Adults with common variable immunodeficiency: a single-center experience. Turk J Med Sci. 2017; 47(1): 1-12.

15. NOBRE FA, et al. Impacto do tratamento com imunoglobulina humana intravenosa no número de pneumonias em pacientes com deficiência de anticorpo. Revista Brasileira de Alergia e Imunopatologia. 2012 ; 35 (1): 25-29.

16. PATEL $S$, et al. Interstitial lung disease in patients with common variable immunodeficiency disorders: several different pathologies? Clin Exp Immunol. 2019; 198(2): 212-223.

17. STIEHM ER. Adverse effects of human immunoglobulin therapy. Transfus Med Rev. 2013; 27(3): 171-8.

18. TRINCÃO DP, et al. Imunodeficiência Comum Variável: A Propósito da Importância da Referenciação a Centros Especializados. Cartas ao Editor, Acta Med Port, 2020; 33(2): 150-153.

19. YAZDANI R, et al. Common Variable Immunodeficiency: Epidemiology, Pathogenesis, Clinical Manifestations, Diagnosis, Classification, and Management. J Investig Allergol Clin Immunol. 2020; 3 (1): 14-34.

20. ZEA-VERA AF, AGUDELO-ROJAS OL. Disseminated bronchiectasis in an adult with common variable immunodeficiency. Colomb. Med., Cali, 2015; 46(1): 47-50. 